Tim Leiner

Christoph U. Herborn

Mathias Goyen

Published online: 26 April 2007

(C) Springer-Verlag 2007

T. Leiner $(\bowtie)$

Department of Radiology,

Maastricht University Hospital,

Peter Debijelaan 25,

6229HX Maastricht, The Netherlands

e-mail: leiner@rad.unimaas.nl

T. Leiner

Cardiovascular Research Institute

Maastricht (CARIM),

Maastricht University,

Maastricht, The Netherlands

C. U. Herborn - M. Goyen

Medical Prevention Center Hamburg,

University Medical Center

Hamburg-Eppendorf,

Martinistrasse 52,

20246 Hamburg, Germany

\title{
Nephrogenic systemic fibrosis is not exclusively associated with gadodiamide
}

The subject of nephrogenic systemic fibrosis (NSF), first addressed in the radiological community in this journal a few months ago [1], is a fast-moving topic with new details arising almost every day.

NSF is a rare, debilitating, and sometimes fatal condition that only occurs in patients with severe renal impairment. It is characterized by the formation of connective tissue in the skin, which becomes thickened, coarse, and hard, sometimes leading to contractures and joint immobility.

NSF has been associated with use of certain gadolinium $(\mathrm{Gd})$-containing magnetic resonance imaging contrast agents in patients with severe renal impairment [2, 3]. Since its recognition in 1997 by Dr. Sean E. Cowper, a dermatopathologist of Yale University [2], more than 215 cases have been recorded [4]. Recent reports by Grobner [5] and Marckmann et al. [7] were among the first to suggest a link with the Gd-containing contrast agent gadodiamide (Omniscan ${ }^{\circledR}$, Gd-DTPABMA, GE Healthcare, Buckinghamshire, UK).

Subsequent to these reports, the United States Food and Drug Administration (FDA) issued a public health advisory in June 2006 recommending that Gd-containing contrast agents be used only if clearly necessary in patients with advanced kidney failure and that prompt dialysis is instituted in patients with advanced kidney failure who receive $\mathrm{Gd}$-containing contrast agents [8]. In January of 2007, this was followed by an announcement of the pharmacovigilance working party of the European Medicines Agency (EMEA) who decided that it is contraindicated to use gadodiamide in patients with a glomerular filtration rate (GFR) below $30 \mathrm{ml} / \mathrm{min}$, on dialysis, and patients who have undergone liver transplantation. Due to immature kidney function in neonates and infants up to one of age, gadodiamide should only be used in these patients after careful consideration [9]. At present, there is no established treatment for NSF although a number of therapies have been used with varying success. For a more detailed description of the clinical manifestations of NSF and the role of gadodiamide, we refer the Web site of the International Center for Nephrogenic Fibrosing Dermopathy Research [3] and the previous editorial on this subject by Thomsen [1].

Although the exact mechanism by which NSF develops remains elusive, recent publications have shed further light on the pathogenesis of NSF. Grobner found that patients with renal disease who were exposed to gadodiamide and did develop NSF were acidotic at the time of imaging, while those that did not develop NSF were not acidotic [5]. In a forthcoming publication on NSF in 1816 patients undergoing hemodialysis, Collidge et al. found a positive association between the cumulative dose of gadodiamide and the development of NSF. Patients with nephrogenic systemic fibrosis received a higher median cumulative dose of Gadodiamide $(0.39 \mathrm{mmol} / \mathrm{kg}$ v $0.23 \mathrm{mmol} / \mathrm{kg}$, $\mathrm{p}=0.008)$ and received more gadolin- 
ium-enhanced magnetic resonance imaging than their nonaffected, gadolinium-exposed counterparts [6]. Furthermore, as the release of free $\mathrm{Gd}^{3+}$ through a process of transmetallation in patients with reduced elimination of Gd-containing contrast agents via the kidneys is considered to be a possible trigger for NSF, the stability of the $\mathrm{Gd}-$ chelate complexes is an important factor in the overall risk assessment across the various contrast agents. Based on their chemical structures, Gd-containing contrast agents may be divided into two groups: linear (open-chain) chelates and macrocyclic chelates. A further subdivision is made between ionic and nonionic linear chelates [10]. Nonionic linear chelates such as gadodiamide display clearly lower stability values than ionic linear chelates and are characterized by a comparably high concentration of excess free ligand in the formulation [10]. The ionic linear chelates such as Gd-diethylenetriamine penta-acetic acid (DTPA) are characterized by a much higher complex stability. Macrocyclic chelates differ significantly from linear chelates regarding the kinetics of complexation and decomplexation. Significant activation energy is necessary to both generate and dissociate the Gd complexes, also resulting in high complex stability [10].

Two very recent publications confirm the biological relevance of the difference between macrocyclic and ionic linear vs nonionic linear chelates. Boyd et al. [11] have now convincingly demonstrated the presence of $\mathrm{Gd}$ in areas of calcium phosphate deposition in blood vessels in a skin biopsy obtained from a patient with clinical features consistent with NSF after administration of gadodiamide. These results were corroborated in a report by High et al. [4] who studied tissue from seven patients with clinical and histopathological diagnosis of NSF after gadodiamide administration. Gadolinium was detected in the histological specimens of four of these seven patients. In all cases, the Gd particles appeared to be intracellular, possibly in the lysosomes [4].

Although the overwhelming majority of known NSF cases at this point represent patients who have been given gadodiamide, the present update was motivated by the fact that it has now become clear that other Gd-containing contrast agents are also associated with NSF. In a recent editorial in Radiology, Kuo et al. [12] report on the 57 cases of NSF reported to the FDA. Forty-three cases of NSF were associated with gadodiamide, six cases with gadopentetate dimeglumine (Magnevist ${ }^{\circledR}$; Bayer Schering Pharma AG, Berlin, Germany), two with gadoversetamide [OptiMARK®, Mallinckrodt, St. Louis, MO, USA (presently not available in Europe)], and three with both gadodiamide and gadoversetamide. In three cases, the associated Gdbased contrast agent was not established [12]. In a series of 13 biopsy-proven patients with NSF, Sadowski et al. [13] also report on a case associated with both gadodiamide and gadobenate dimeglumine (MultiHance ${ }$, Bracco Diagnostics, Milan, Italy), given 1 week apart.
On the basis of spontaneous reporting of adverse drug reactions, a total of 120 cases of NSF in association with the administration of Omniscan ${ }^{\circledR}$ were reported by GE Healthcare as of March 2007 [14]. A total of 42 reports of NSF in association with gadopentetate dimeglumine have been reported to Bayer Schering Pharma AG from worldwide sources as of March 23, 2007. Twenty-one of these cases were reported by a single center [15]. Onset of NSF symptoms in all available reports dates back up to several years. The time span between administration of MR contrast medium and occurrence of symptoms ranges between several days and several years. Of the total of 42 cases, 16 were assessed as possibly related, 3 were assessed as unlikely related, and 23 were assessed as unclassifiable, meaning that the information provided was not sufficient to verify the diagnosis of NSF by deep skin biopsy and histopathology and/or to link the cases exclusively to the administration of gadopentetate dimeglumine.

At present, there are no known cases of NSF associated with administration of other Gd-containing contrast agents on the market in Europe: gadobutrol (Gadovist ${ }^{\circledR}$ Bayer Schering Pharma AG), gadofosveset trisodium (Vasovist ${ }^{\circledR}$, Bayer Schering Pharma AG), gadoxetic acid (Primovist ${ }^{\circ}$,

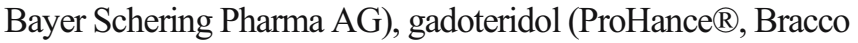
Diagnostics), and gadoterate meglumine (Dotarem $\AA$, Guerbet S.A., Aulnay-sur-Bois, France).

Despite the fact that, at present, not all Gd-based contrast agents have been associated with NSF, it is paramount that these agents be used with caution in patients with renal insufficiency, especially considering the fact that the pathogenesis has not definitively been established. Reflecting these concerns, the Committee for Medicinal Products for Human Use of the EMEA has requested the marketing authorization holders of MR contrast agents to introduce a warning in the summary of product characteristics ("package insert") about the occurrence of NSF in patients with severe renal impairment.

How do these recent findings influence clinical practice? Based on the approach by Kuo et al. [12], published in the March 2007 issue of Radiology, we recommend the following approach for patients with stage 4 or 5 chronic kidney disease (i.e., patients on peritoneal dialysis or hemodialysis) or patients with a GFR of less than $30 \mathrm{ml} \mathrm{min}^{-1} 1.73 \mathrm{~m}^{-2}$ :

1. In consultation with the ordering physician, we consider ultrasound, CT, and non-contrast-enhanced MR angiography as alternative imaging modalities

2. We do not recommend administering gadodiamide to a patient with any history of renal disease. We only administer Gd-based MR contrast agents to a patient with renal failure if the patient subsequently undergoes dialysis. If administration of a Gd-based MR contrast agent is deemed necessary, we consider using the lowest dose needed to reliably provide the diagnostic information being clinically sought. If there is a 
diagnosis or clinical suspicion of NSF in the patient, we discourage exposure to any Gd chelates.

3. For patients maintained with hemodialysis, we ensure hemodialysis treatment as soon as possible, ideally within $3 \mathrm{~h}$ after the administration of the Gd-containing contrast agent. A second dialysis session within $24 \mathrm{~h}$ can also be performed if it is clinically safe to do so. For patients undergoing peritoneal dialysis, we ensure that patients have no periods with a dry abdomen (i.e., peritoneal cavity contains no dialysate), and we perform more frequent manual exchanges or additional automated peritoneal dialysis cycles for at least $48 \mathrm{~h}$ after administration.

At this time, the relationship between NSF and Gd chelates remains unclear. Further studies are now underway at the Centers for Disease Control Prevention, the FDA, and in the medical regulatory agencies of the European Union. If a patient with NSF is encountered, the following should be done [12]:
1. A history of administration of a Gd-based MR contrast agent in the weeks or months preceding the initial diagnosis should be ascertained. In addition, the date of administration, the dose and brand of the contrast agent administered, as well as the date of onset or diagnosis of NSF should be noted.

2. The event should be reported to the EMEA (http:// www.emea.europa.eu) and FDA online through the MedWatch reporting program (http://www.fda.gov/ medwatch/) or by phone (1-800-FDA-1088) or appropriate non-E.U./non-US regulatory agencies.

3. The case should be reported to the NSF registry at Yale University (http://www.icnfdr.org) and to the European Society of Urogenital Radiology (http://www.esur.org).

\section{References}

1. Thomsen H (2006) Nephrogenic systemic fibrosis: a serious late adverse reaction to gadodiamide. Eur Radiol 16:2619-2621

2. Cowper SE, Robin HS, Steinberg SM, Su LD, Gupta S, LeBoit PE (2000) Scleromyxoedema-like cutaneous diseases in renal-dialysis patients. Lancet 356:1000-1001

3. Cowper SE (2007) Nephrogenic fibrosing dermopathy (NFD/NSF Web site). 2001-2007. Available at http://www. icnfdr.org. Accessed March 22, 2007.

4. High WA, Ayers RA, Chandler J, Zito $\mathrm{G}$, Cowper SE (2007) Gadolinium is detectable within the tissue of patients with nephrogenic systemic fibrosis. J Am Acad Dermatol 56:21-26

5. Grobner T (2006) Gadolinium - a specific trigger for the development of nephrogenic fibrosing dermopathy and nephrogenic systemic fibrosis? Nephrol Dial Transplant 21:1104-1108

6. Collidge TA, Thompson PC, Mark PB, Traynor JP, Jardine A, Morris STW, Simpson K, Roditi G (2007) Gadolinium-enhanced magnetic resonance imaging and nephrogenic systemic fibrosis - a retrospective case-control Study. Radiology (in press)
7. Marckmann P, Skov L, Rossen K, Dupont A, Damholt MB, Heaf JG, Thomsen HS (2006) Nephrogenic systemic fibrosis: suspected causative role of gadodiamide used for contrast-enhanced magnetic resonance imaging. J Am Soc Nephrol 17:2359-2362

8. United States Food and Drug Administration, Rockville, MD, USA. Public Health Advisory. Gadolinium-containing contrast agents for magnetic resonance imaging (MRI): Omniscan, OptiMARK, Magnevist, ProHance and Multihance. Available from URL: http://www.fda.gov/cder/drug/advisory/ gadolinium_agents.htm. (Accessed March 22, 2007)

9. European Society for Magnetic Resonance in Medicine and Biology. Vienna, Austria. Available from URL: http://www.esmrmb.org/index.php? pid $=587 \& \mathrm{SID}=5 \mathrm{bbbc5f75411 \textrm {bab }}$ 76e4aee221e8eacdf. (Accessed March $30,2007)$

10. Idée J-M, Port M, Raynal I, Schaefer M, Le Greneur S, Corot C (2006) Clinical and biological consequences of transmetallation induced by contrast agents for magnetic resonance imaging: a review. Fundam Clin Pharmacol 20:563-576
11. Boyd AS, Zic JA, Abraham JL (2007) Gadolinium deposition in nephrogenic fibrosing dermopathy. J Am Acad Dermatol 56:27-30

12. Kuo PH, Kanal E, Abu-Alfa AK, Cowper SE (2007) Gadolinium-based MR contrast agents and nephrogenic systemic fibrosis. Radiology 242:647649

13. Sadowski EA, Bennett LK, Chan MR, Wentland AL, Garrett AL, Garret RW, Djamali A (2007) Nephrogenic systemic fibrosis: risk factors and incidence estimation. Radiology 242 (3):647-649

14. GE Healthcare, Buckinghamshire, United Kingdom. GE Healthcare paper on nephrogenic systemic fibrosis, March 2007. Available from URL: http://www.amershamhealth-us.com/ omniscan/GE\%20Healthcare\%20Paper $\% 200 n \% 20$ Nephrogenic $\% 20$ Systemic \%20Fibrosis.pdf. (Accessed March 30, 2007)

15. Bayer Schering Pharma AG, Berlin, Germany. NSF/NFD Update. Available from URL: http://www.berleximaging. $\mathrm{com} / \mathrm{html} /$ magnevist $/ \mathrm{nsf}$ nfd.html. (Accessed March 23, 2007) 